\title{
Single-center open-label randomized study of anemia management improvement in ESRD patients with secondary hyperparathyroidism
}

\author{
Antonio Bellasi ${ }^{1,2}$, Stefano Mangano ${ }^{1}$, Claudio Minoretti ${ }^{1}$, Carlo Campana ${ }^{2}$, Biagio Di lorio ${ }^{3}$, Luca Di Lullo ${ }^{4}$, Carlo Ratti ${ }^{1}$, \\ Mario Cozzolino ${ }^{2}$ \\ ${ }^{1}$ Department of Nephrology and Dialysis, Saint Anne's Hospital, Como - Italy \\ ${ }^{2}$ Department of Health Sciences, University of Milan, Milan - Italy \\ ${ }^{3}$ Department of Cardiology, Saint Anne's Hospital, Como - Italy \\ ${ }^{4}$ Department of Nephrology and Dialysis, PO "A Landolfi", Solofra (Avellino) - Italy
}

\begin{abstract}
Background: Whether anemia and mineral bone abnormalities (chronic kidney disease-mineral bone disorder [CKD-MBD]) are associated still remains to be elucidated. Both anemia and CKD-MBD have been associated with adverse cardiovascular outcome and poor quality of life. However, recent evidence suggests that use of large doses of erythropoietin-stimulating agents (ESAs) to correct hemoglobin ( $\mathrm{Hb}$ ) may be detrimental in CKD. The Optimal Anemia Treatment in End Stage Renal Disease (ESRD) (Optimal ESRD Treatment) study will assess whether lowering of parathyroid hormone (PTH) is associated with a reduction in ESA consumption.

Methods: The Optimal ESRD Treatment study is a pilot single-center open-label study with blinded end point (a prospective randomized open blinded end-point [PROBE] design) enrolling 50 patients on maintenance dialysis. Eligible patients with intact PTH (iPTH) $300-540 \mathrm{pg} / \mathrm{mL}$ and $\mathrm{Hb} 10-11.5 \mathrm{~g} / \mathrm{dL}$ will be randomized $1: 1$ to strict PTH control (150-300 pg/mL) versus standard care (PTH range 300-540 pg/mL). Available drugs for CKD-MBD and anemia treatment will be managed by the attending physician to maintain the desired levels of PTH (according to study arm allocation) and $\mathrm{Hb}(10-11.5 \mathrm{~g} / \mathrm{dL})$. Echocardiographic data for cardiac structure and function as well as arterial stiffness will be assessed at study inception and completion.

Conclusions: The Optimal ESRD Treatment study should shed light on the complicated interplay of anemia and CKD-MBD and on the feasibility of clinical trials in this domain. The study results are expected in the spring of 2017.
\end{abstract}

Keywords: Anemia, CKD-MBD, ESA, Valvular calcification

\section{Introduction}

Anemia is one of the most worrisome complications of chronic kidney disease (CKD). Numerous prospective studies have repeatedly documented an increased risk of morbidity and mortality associated with lower levels of hemoglobin (Hb) (1-5). Hence the international guidelines on patient care suggest the use of erythropoietin-stimulating agents (ESAs), iron and folate supplementation for anemia correction in CKD individuals (1-5).

\author{
Accepted: March 9, 2016 \\ Published online: April 26, 2016 \\ Corresponding author: \\ Antonio Bellasi, MD \\ Department of Nephrology and Dialysis \\ Via Ravona 20 \\ 22020 San Fermo Della Battaglia (CO), Italy \\ antoniobellasi@gmail.com
}

However, recent randomized controlled trials (RCTs) have demonstrated that $\mathrm{Hb}$ correction to normal levels increases the risk of major cardiovascular (CV) events (1-5). Although the reasons are still unclear, the cumulative ESA dose and rapid $\mathrm{Hb}$ fluctuations may at least partly explain these findings (6-10). Hence, limiting ESA to the minimal dose needed to achieve the suggested $\mathrm{Hb}$ targets in end-stage renal disease (ESRD) as well as identifying factors associated with ESA response seems advisable (6-10).

Among other factors, mineral metabolism abnormalities (chronic kidney disease-mineral bone disorder [CKD-MBD]) have repeatedly been associated with poor outcomes and have emerged as a risk factor for severe anemia as well as for use of large ESA doses (11-13). In this regard, a few observational studies have suggested a linear inverse association between parathyroid hormone (PTH) and ESA dose in ESRD patients $(11,12)$, although these findings may be the result of a faster deterioration of the CV system associated with higher PTH levels (14).

Due to the lack of RCTs that clearly demonstrate any survival benefit associated with strict PTH control, the latest 
Kidney Disease: Improving Global Outcomes (KDIGO) guidelines on CKD-MBD management suggest a higher reference target for intact parathyroid hormone (iPTH) (2-9 times the upper level of the normal range) when compared with the National Kidney Foundation (NKF) guidelines published in $2003(150-300 \mathrm{pg} / \mathrm{mL})(14)$.

The aim of the Optimal Anemia Treatment in End Stage Renal Disease (ESRD) (Optimal ESRD Treatment) study is to test whether a tighter PTH control is associated with an ESA dose reduction and a slower $\mathrm{CV}$ system deterioration in ESRD patients receiving dialysis.

\section{Methods}

The Optimal ESRD Treatment study is a pilot, pragmatic, single-center, open-label trial with a blinded end point (prospective randomized open blinded end-point [PROBE] design) aimed at improving patient care. The study protocol was reviewed and approved by the local ethics committee of Azienda Ospedaliera Sant'Anna, Como (Parere Unico 61/13, May 16, 2013) and was registered at https://clinicaltrials.gov/ (NCT02273570), with the official title "Single-center, Open-label, Randomized Study of Anemia Management Improvement in End Stage Renal Disease (ESRD) Patients With Secondary Hyperparathyroidism." All procedures involved in the study will be conducted in adherence to the Declaration of Helsinki and the Ethical Principles for Medical Research Involving Human Subjects, and according to the principles of Good Clinical Practice. lows:

The Optimal ESRD Treatment study objectives are as fol-

1. Primary objective: to test whether a tighter PTH control (PTH level $<300 \mathrm{pg} / \mathrm{mL}$ vs. PTH level between 300 and $540 \mathrm{pg} / \mathrm{mL}$ ) is associated with a lower ESA dose use to achieve the target $\mathrm{Hb}$ of $10.0-11.5 \mathrm{~g} / \mathrm{dL}$.

2. Secondary objective: to test whether a tighter PTH control to achieve a PTH level $<300 \mathrm{pg} / \mathrm{mL}$ vs. PTH level between 300 and $540 \mathrm{pg} / \mathrm{mL}$ is associated with a better iron storage mobilization.

3. Secondary objective: to test whether a tighter PTH control to achieve a PTH level $<300 \mathrm{pg} / \mathrm{mL}$ vs. PTH level between 300 and $540 \mathrm{pg} / \mathrm{mL}$ is associated with cardiac valve deposition and progression attenuation (assessed via echocardiogram).

4. Secondary objective: to test whether a tighter PTH control to achieve a PTH level $<300 \mathrm{pg} / \mathrm{mL}$ vs. PTH level between 300 and $540 \mathrm{pg} / \mathrm{mL}$ is associated with an increase in arterial stiffness attenuation (assessed via applanation tonometry).

5. Secondary objective: to test whether a tighter PTH control to achieve a PTH level $<300 \mathrm{pg} / \mathrm{mL}$ vs. PTH level between 300 and $540 \mathrm{pg} / \mathrm{mL}$ is associated with a better CKD-MBD control.

Eligible patients should be adult men and women (i.e., age $>18$ years), on thrice-weekly maintenance dialysis via arteriovenous fistula. They should be undergoing treatment with an ESA with an $\mathrm{Hb}$ level at study entry between 10.0 and $11.5 \mathrm{~g} / \mathrm{dL}$, iPTH between 300 and $600 \mathrm{pg} / \mathrm{mL}$ and $\mathrm{Kt} / \mathrm{V}>1.2$.
Finally, all study participants should able to provide a signed informed consent prior to the initiation of the study.

All eligible patients will be randomized (1:1) to one of the study arms in which a different PTH control will be enforced during the 12 months of study follow-up:

1. Control group (standard care group): the iPTH target in this group is $300-540 \mathrm{pg} / \mathrm{mL}$.

2. Optimal CKD-MBD control group: in this group, the PTH target is $150-300 \mathrm{pg} / \mathrm{mL}$, to be achieved with a therapeutic algorithm.

To achieve the desired PTH (according to study arm allocation) and $\mathrm{Hb}(10.0-11.5 \mathrm{~g} / \mathrm{dL}$ ) levels, no specific therapeutic algorithm is to be enforced, and the attending physician will be responsible for prescriptions as well as dose titration of the different drugs available on the market for CKD-MBD and anemia management.

To detect meaningful changes in $\mathrm{CV}$ structure and function associated with levels of iPTH, the presence of cardiac valve calcification, cardiac function and arterial stiffness will be assessed noninvasively via transthoracic echocardiogram and applanation tonometry at study entry as well as study completion (Tab. I summarizes the Optimal ESRD Treatment study checklist). Briefly, a standard transthoracic echocardiogram will be performed. Parasternal short and long axes as well as 4- and 5-chamber views will be acquired to assess cardiac structure and function. In particular, the presence and extension of cardiac valve calcification will be scored as suggested by Corciu and collaborators (15). Arterial stiffness and thoracic aorta blood pressure will be evaluated noninvasively and expressed as pulse wave velocity, thoracic aorta central blood pressure and augmentation index. To reduce the intra- and inter-reader variability, echocardiogram and applanation tonometry will be performed before the midweek dialysis session, and echocardiogram readings will be centralized.

It was anticipated that the entire study should take about 18 months (from first patient in to last patient out): 6-month recruitment phase and 12-month active treatment period. Active patient recruitment began in March 2015. However, as of September 2015, only 17 patients had been randomized, due to a slower than anticipated recruitment rate, and it is now anticipated that the study will last until February 2017.

The Optimal ESRD Treatment study is funded by an unrestricted research grant from Amgen and from the Italian Society of Nephrology.

\section{Statistical analysis and sample size estimation}

Standard descriptive statistics as well as parametric and nonparametric tests will be used. Linear regression models will be adopted to test the difference in ESA use, cardiac valve calcification and arterial stiffness between groups at study completion. All efficacy analyses will be performed on an intention-to-treat basis.

According to the study protocol, the primary end point is defined as percentage reduction in weekly ESA consumption to maintain $\mathrm{Hb}$ levels within the recommended range $10.0-11.5 \mathrm{~g} / \mathrm{dL}$. 
TABLE I - Optimal ESRD Treatment study checklist

\begin{tabular}{|c|c|c|c|c|c|c|c|c|c|c|c|c|}
\hline Month & 1 & 2 & 3 & 4 & 5 & 6 & 7 & 8 & 9 & 10 & 11 & 12 \\
\hline Patient history & $x$ & & & & & & & & & & & \\
\hline Concomitant medication & $x$ & $x$ & $x$ & $x$ & $x$ & $x$ & $x$ & $x$ & $x$ & $x$ & $x$ & $x$ \\
\hline Physical examination & $x$ & $x$ & $x$ & $x$ & $x$ & $x$ & $x$ & $x$ & $x$ & $x$ & $x$ & $x$ \\
\hline ECG & $x$ & & & & & & & & & & & $x$ \\
\hline Biobank & $x$ & & & & & & & & & & & $x$ \\
\hline Lab tests* & $x$ & $x$ & $x$ & $x$ & $x$ & $x$ & $x$ & $x$ & $x$ & $x$ & $x$ & $x$ \\
\hline Cardiac valve calcification assessment & $x$ & & & & & & & & & & & $x$ \\
\hline Safety assessment & $x$ & $x$ & $x$ & $x$ & $x$ & $x$ & $x$ & $x$ & $x$ & $x$ & $x$ & $x$ \\
\hline
\end{tabular}

ECG = echocardiography; iPTH = intact parathyroid hormone

* As per standard practice.

The following secondary end points will also be investigated:

- Change in iron status and storage, defined as change in iron consumption per week, percentage of patients with transferrin saturation $>20 \%$ between groups at study completion, change in hepcidin levels between groups at study completion. These end points will be compared between and within treatment groups.

- Difference (absolute change and percentage increase) in prevalence of valvular calcification deposition and progression as detected by echocardiography between groups. This end point will be compared between and within treatment groups.

- Difference (absolute change and percentage increase) in pulse wave velocity as assessed by applanation tonometry between groups. This end point will be compared between and within treatment groups.

Given that that this is a pilot study and there are no data for an accurate sample size estimation, and assuming: (i) $40 \%$ ESA dose reduction in the Optimal CKD-MBD control group, (ii) power $80 \%$, (iii) statistical significance $5 \%$ and (iv) dropout rate of $10 \%$, it is estimated that 50 patients ( $n=25$ per study arm) are needed.

\section{Discussion}

Observational studies suggest a close association between CKD-MBD and anemia. Nonetheless, no RCT has investigated whether CKD-MBD manipulation may ameliorate anemia control in ESRD (11-13). The aim of the Optimal ESRD Treatment study is to test whether a strict PTH control (i.e., $150-300 \mathrm{pg} / \mathrm{mL}$ vs. $300-540 \mathrm{pg} / \mathrm{mL}$ ) reduces ESA consumption in a cohort of 50 patients on maintenance dialysis. The secondary objectives of the Optimal ESRD Treatment study are to investigate the impact on the CV system, of 2 different iPTH targets within the suggested range of twofold to ninefold the upper level of the laboratory reference for iPTH (14).

The Optimal ESRD Treatment study is a pragmatic trial. Indeed, with the exception of a vitamin D usage limitation (i.e., the cumulative weekly dose of paricalcitol or equivalent should not exceed $6 \mu \mathrm{g}$ ), no specific recommendations on drug use to achieve the desired levels of iPTH and $\mathrm{Hb}$ are to be enforced. The attending physician is free to prescribe and adjust doses of commonly available drugs for CKD-MBD and anemia management.

Although patient recruitment has been slower than expected and a protocol amendment is under evaluation to not limit patient eligibility to individuals with arteriovenous fistula as vascular access, it is anticipated that the study will be completed and the study results available in spring 2017.

The single-center design and small sample size are indeed limitations of this pilot study. Nonetheless, the Optimal ESRD Treatment study will shed some light on the feasibility of future larger studies in this domain. Far more importantly, it will provide some hints for how to optimize ESRD patient care. An ongoing, large, properly powered, multicenter study is testing the hypothesis that ESA dose is linked to poor outcome in ESRD (16). Knowing what factors are associated with increased use of ESA and knowing that manipulation of bone mineral abnormalities may result in ESA use or dose minimization will ultimately result in improved patient care. Although only 50 patients will participate in the study, the fact that all study participants receive maintenance dialysis in a single center, as well as the centralization of laboratory and imaging procedures, should minimize the between-center 
and inter-reader variability, thus increasing the chance to detect a meaningful result if it exists.

In summary, the Optimal ESRD Treatment study aims to shed light on any causative association between PTH and anemia. The study results are expected for spring 2017, and it is the authors' opinion that they will be valuable for improved patient care and/or for future larger studies to be undertaken in this domain.

\section{Disclosures}

Financial support: The Optimal ESRD Treatment study is funded by an unrestricted research grant from Amgen and from the Italian Society of Nephrology.

Conflict of interest: None of the authors has any financial interest related to this study to disclose.

\section{References}

1. National Kidney Foundation. KDOQI clinical practice guidelines and clinical practice recommendations for anemia in chronic kidney disease: 2007 update of hemoglobin target. Am J Kidney Dis. 2007;50:471-530.

2. National Kidney Foundation. K/DOQI clinical practice guidelines for bone metabolism and disease in chronic kidney disease. Am J Kidney Dis. 2003;42:S1-S201.

3. Locatelli F, Nissenson AR, Barrett BJ, et al. Clinical practice guidelines for anemia in chronic kidney disease: problems and solutions. A position statement from Kidney Disease: Improving Global Outcomes (KDIGO). Kidney Int. 2008;74:1237-1240.

4. Locatelli F, Pisoni RL, Akizawa T, et al. Anemia management for hemodialysis patients: Kidney Disease Outcomes Quality Initiative (K/DOQI) guidelines and Dialysis Outcomes and Practice Patterns Study (DOPPS) findings. Am J Kidney Dis. 2004;44: 27-33.

5. Pisoni RL, Bragg-Gresham JL, Young EW, et al. Anemia management and outcomes from 12 countries in the Dialysis Outcomes and Practice Patterns Study (DOPPS). Am J Kidney Dis. 2004;44:94-111.

6. Regidor DL, Kopple JD, Kovesdy CP, et al. Associations between changes in hemoglobin and administered erythropoiesis-stim- ulating agent and survival in hemodialysis patients. J Am Soc Nephrol. 2006;17:1181-1191.

7. Lacson E Jr, Ofsthun N, Lazarus JM. Effect of variability in anemia management on hemoglobin outcomes in ESRD. Am J Kidney Dis. 2003;41:111-124.

8. Gilbertson DT, Ebben JP, Foley RN, Weinhandl ED, Bradbury BD, Collins AJ. Hemoglobin level variability: associations with mortality. Clin J Am Soc Nephrol. 2008;3:133-138.

9. Fishbane S, Berns JS. Hemoglobin cycling in hemodialysis patients treated with recombinant human erythropoietin. Kidney Int. 2005;68:1337-1343.

10. Ebben JP, Gilbertson DT, Foley RN, Collins AJ. Hemoglobin level variability: associations with comorbidity, intercurrent events, and hospitalizations. Clin J Am Soc Nephrol. 2006; 1:1205-1210.

11. Fusaro M, D'Angelo A, Naso A, et al. Treatment with calcimimetic (cinacalcet) alters epoetin dosage requirements in dialysis patients: preliminary report. Ren Fail. 2011;33:732-735.

12. Battistella M, Richardson RM, Bargman JM, Chan CT. Improved parathyroid hormone control by cinacalcet is associated with reduction in darbepoetin requirement in patients with endstage renal disease. Clin Nephrol. 2011;76:99-103.

13. Capuano A, Serio V, Pota A, Memoli B, Andreucci VE. Beneficial effects of better control of secondary hyperparathyroidism with paricalcitol in chronic dialysis patients. J Nephrol. 2009; 22:59-68.

14. Kidney Disease Improving Global Outcomes (KDIGO) CKDMBD Work Group. KDIGO clinical practice guideline for the diagnosis, evaluation, prevention, and treatment of chronic kidney disease-mineral and bone disorder (CKD-MBD). Kidney Int Suppl. 2009;76:S1-S130.

15. Corciu A, Siciliano V, Poggianti E, Petersen C, Venneri L, Picano E. Cardiac calcification by transthoracic echocardiography in patients with known or suspected coronary artery disease. Int J Cardiol. 2010;23;142(3):288-295.

16. Strippoli GF; Clinical Evaluation of the Dose of Erythropoietins Study Group (C.E. DOSE). Effects of the dose of erythropoiesis stimulating agents on cardiovascular events, quality of life, and health-related costs in hemodialysis patients: the Clinical Evaluation of the Dose of Erythropoietins (C.E. DOSE) trial protocol. Trials. 2010;11:70. 